\title{
OPE for B-meson distribution amplitude and dimension-5 HQET operators
}

\author{
Hiroyuki Kawamura* \\ Department of Mathematical Sciences, University of Liverpool, Liverpool, L69 3BX, United \\ Kingdom \\ E-mail: Hiroyuki.Kawamura@liverpool.ac.uk
}

\section{Kazuhiro Tanaka}

Department of Physics, Juntendo University, Inba, Chiba 270-1695, Japan

E-mail: tanakakesakura.juntendo.ac.jp

The $B$-meson light-cone distribution amplitude (LCDA) is defined as the matrix element of a quark-antiquark bilocal light-cone operator in the heavy-quark effective theory (HQET) and is a building block of QCD factorization formula for exclusive $B$-meson decays. When the corresponding bilocal HQET operator has a light-like distance $t$ between the quark and antiquark fields, the scale $\sim 1 / t$ separates the UV and IR regions, which induce the cusp singularity in radiative corrections and the mixing of multiparticle states in nonperturbative corrections, respectively. We treat the bilocal HQET operator based on the operator product expansion (OPE), disentangling the singularities from the IR and UV regions systematically. The matching at the next-to-leading order $\alpha_{s}$ is performed in the $\overline{\mathrm{MS}}$ scheme with a complete set of local operators of dimension $d \leq 5$, through a manifestly gauge-invariant calculation organizing all contributions in the coordinate space. The result exhibits the Wilson coefficients with Sudakov-type double logarithms and the higher-dimensional operators with additional gluons. This OPE yields the $B$-meson LCDA for $t$ less than $\sim 1 \mathrm{GeV}^{-1}$, in terms of $\bar{\Lambda}=m_{B}-m_{b}$ and the two additional HQET parameters as matrix elements of dimension-5 operators. The impact of these novel HQET parameters on the integral relevant to exclusive $B$ decays, $\lambda_{B}$, is also discussed.

International Workshop on Effective Field Theories: from the pion to the upsilon February 2-6 2009

Valencia, Spain

\footnotetext{
* Supported in part by the UK Science \& Technology Facilities Council under grant number PP/E007414/1.

$\dagger$ Speaker.

${ }^{\ddagger}$ Supported by the Grant-in-Aid for Scientific Research No. B-19340063.
} 
For the exclusive $B$-meson decays, such as $B \rightarrow \pi \pi, \rho \gamma, \ldots$, systematic methods have been developed using QCD factorization based on the heavy-quark limit [1-3]. In the corresponding factorization formula of the decay amplitude, essential roles are played by the light-cone distribution amplitudes (LCDAs) for the participating mesons, which include nonperturbative long-distance contributions. In particular, in addition to the LCDAs for the light mesons $\pi, \rho$, etc., produced in the final state, the LCDA $\tilde{\phi}_{+}$for the $B$ meson, defined as the vacuum-to-meson matrix element [4],

$$
\tilde{\phi}_{+}(t, \mu)=\frac{1}{i F(\mu)}\left\langle 0\left|\bar{q}(t n) \mathrm{P} e^{i g \int_{0}^{t} d \lambda n \cdot A(\lambda n)} h \gamma_{5} h_{v}(0)\right| \bar{B}(v)\right\rangle=\int d \omega e^{-i \omega t} \phi_{+}(\omega, \mu),
$$

also participates in processes where large momentum is transferred to the soft spectator quark via hard gluon exchange [1-3]. Here, the bilocal operator is built of the $b$-quark and light-antiquark fields, $h_{v}(0)$ and $\bar{q}(t n)$, linked by the Wilson line at a light-like separation $t n$, with $n_{\mu}$ as the lightlike vector $\left(n^{2}=0, n \cdot v=1\right)$, and $v_{\mu}$ representing the 4-velocity of the $B$ meson; a difference between (1) and the familiar pion-LCDA is that $h_{v}(0)$ is an effective field in the heavy-quark effective theory (HQET). $\mu$ denotes the scale where the operator is renormalized, and $F(\mu)$ is the decay constant in HQET, $F(\mu)=-i\left\langle 0\left|\bar{q} h \gamma_{5} h_{v}\right| \bar{B}(v)\right\rangle$. The RHS in (1) defines the momentum representation, with $\omega v^{+}$denoting the LC component of the momentum of the light antiquark.

The "IR structure" of (1), studied using constraints from the equations of motion (EOM) and heavy-quark symmetry [5], as well as the "UV structure", calculated in the 1-loop renormalization of the bilocal operator in (1) [6], is notoriously peculiar compared with the pion LCDA. For a full description of (1) which would involve a complicated mixture of the IR and UV structures, we first calculate the radiative corrections, taking into account hard and soft/collinear loops. The one-particle-irreducible 1-loop diagrams (1LDs) for the 2-point function $\left\langle\bar{q}(t n) h \gamma_{5} h_{v}(0)\right\rangle$ of (1) yield [7] $\left(\langle\cdots\rangle \equiv\langle 0|\cdots| \bar{B}(v)\rangle\right.$, the Wilson line is suppressed, and $\left.C_{F}=\left(N_{c}^{2}-1\right) /\left(2 N_{c}\right)\right)$

$$
\begin{aligned}
& 1 \mathrm{LDs}=\frac{\alpha_{s} C_{F}}{2 \pi} \int_{0}^{1} d \xi\left[\left\{-\left(\frac{1}{2 \varepsilon_{U V}^{2}}+\frac{L}{\varepsilon_{U V}}+L^{2}+\frac{5 \pi^{2}}{24}\right) \delta(1-\xi)+\left(\frac{1}{\varepsilon_{U V}}-\frac{1}{\varepsilon_{I R}}\right)\left(\frac{\xi}{1-\xi}\right)_{+}\right.\right. \\
& \left.\left.-\left(\frac{1}{2 \varepsilon_{I R}}+L\right)\right\}\left\langle\bar{q}(\xi t n) h \gamma_{5} h_{v}(0)\right\rangle-t\left(\frac{1}{\varepsilon_{I R}}+2 L-1-\xi\right)\left\langle\bar{q}(\xi t n) v \cdot \overleftarrow{D} h \gamma_{5} h_{v}(0)\right\rangle\right]+\cdots,
\end{aligned}
$$

in $D=4-2 \varepsilon$ dimensions and Feynman gauge, where $L \equiv \ln \left[i(t-i 0) \mu e^{\gamma_{E}}\right]$ with the $\overline{\text { MS scale } \mu}$ and the Euler constant $\gamma_{E}$. The "vertex-type" correction that connects the light-like Wilson line and $\bar{q}(t n)$ in (1) is associated with only the massless degrees of freedom and yields the scaleless loop-integral that gives the term with the "canceling" UV and IR poles, $1 / \varepsilon_{U V}-1 / \varepsilon_{I R}$, and with the "plus"-distribution $(\xi /(1-\xi))_{+}$as the splitting function; this term is identical to the corresponding correction for the case of the pion LCDA. The other terms in (2) have "non-canceling" UV and IR poles: another vertex-type correction around a "cusp" between the two Wilson lines, the light-like Wilson line of (1) and the time-like Wilson line from $h_{v}(0)=\operatorname{Pexp}\left[i g \int_{-\infty}^{0} d \lambda v \cdot A(\lambda v)\right] h_{v}(-\infty v)$, gives the terms proportional to $\delta(1-\xi)$, which contain the double as well as single UV pole, corresponding to the cusp singularity [6]. The "ladder-type" correction, connecting the two quark fields in (1), gives all the remaining terms in (2), which contain the IR poles and are associated with not only the bilocal operator in (1), but also the higher dimensional operators; the ellipses in (2) are expressed by the operators involving two or more additional covariant derivatives.

The renormalized LCDA is obtained by subtracting the UV poles from (2) with the trivial quark self-energy corrections complemented. Here, the term with the plus-distribution $(\xi /(1-$ 
$\xi))_{+}$is analytic (Taylor expandable) at $t=0$, similar to the pion LCDA, but the other terms are not analytic due to the presence of logarithms $L, L^{2}[6,8]$. In particular, the nontrivial dependence of the latter terms on $t \mu$ through $L$ implies that the scale $\sim 1 / t$ separates the UV and IR regions. Thus, we have to use the operator product expansion (OPE) to treat the different UV and IR behaviors simultaneously: the coefficient functions absorb all the singular logarithms, while, for the local operators to absorb the IR poles, we have to take into account many higher dimensional operators. Such OPE with local operators is useful when the separation $t$ is less than the typical distance scale of quantum fluctuation, i.e., when $t \lesssim 1 / \mu$. We note that an OPE for the $B$-meson LCDA (1) was discussed in [9], taking into account the local operators of dimension $d \leq 4$ and the NLO $\left(O\left(\alpha_{s}\right)\right)$ corrections to the corresponding Wilson coefficients in a "cutoff scheme", where an additional momentum cutoff $\Lambda_{U V}\left(\gg \Lambda_{\mathrm{QCD}}\right.$ ) was introduced, and the OPE, in powers of $1 / \Lambda_{U V}$, was derived for the regularized moments, $M_{j}=\int_{0}^{\Lambda_{U V}} d \omega \omega^{j} \phi_{+}(\omega, \mu)$, in particular, for the first two moments with $j=0,1$; note, $M_{j} \rightarrow \infty$ as $\Lambda_{U V} \rightarrow \infty$ [4]. Here, we derive the OPE for (1), taking into account the local operators of dimension $d \leq 5$ and calculating the corresponding Wilson coefficients at NLO accuracy. Following the discussion above, we carry out the calculation for $t \lesssim 1 / \mu$ in the coordinate space and in the $\overline{\mathrm{MS}}$ scheme, so that there is no need to introduce any additional cutoff.

The most complicated task is the reorganization of contributions from (many) Feynman diagrams in terms of the matrix element of gauge-invariant operators including higher dimensional operators, in particular, the three-body operators of dimension 5, such as $\bar{q} G_{\alpha \beta} h \gamma_{5} h_{v}$ with the field strength tensor $G_{\alpha \beta}$ [4, 5]. To derive the NLO Wilson coefficients associated with such operators, we have to compute the 1-loop diagrams for the 3-point function, as well as those for the 2-point function as in (2), where the former diagrams are obtained by attaching the external gluon line to the latter diagrams in all possible ways. We employ the background field method [10], where the background fields represent the nonperturbative long-distance degrees of freedom and satisfy the exact classical EOM. We use the Fock-Schwinger gauge, $x^{\mu} A_{\mu}^{(c)}(x)=0$, for the background gluon field $A_{\mu}^{(c)}$. This gauge condition is solved to give $A_{\mu}^{(c)}(x)=\int_{0}^{1} d u u x^{\beta} G_{\beta \mu}^{(c)}(u x)$ [10], which allows us to reexpress each Feynman diagram in terms of the matrix element of the operators associated with the field strength tensor. Also, this ensures that the Wilson line in (1), as well as the heavy-quark propagator, does not couple directly to the background gluons while a massless quark or gluon propagator couples to them. With the matching in the $\overline{\mathrm{MS}}$ scheme, we obtain [7] the OPE,

$\bar{q}(t n) \mathrm{P} e^{i g \int_{0}^{t} d \lambda n \cdot A(\lambda n)} \not h \gamma_{5} h_{v}(0)=C_{1}^{(3)}(t, \mu) \mathscr{O}_{1}^{(3)}(\mu)+\sum_{k=1}^{2} C_{k}^{(4)}(t, \mu) \mathscr{O}_{k}^{(4)}(\mu)+\sum_{k=1}^{7} C_{k}^{(5)}(t, \mu) \mathscr{O}_{k}^{(5)}(\mu)$,

where the summation is over a basis of local operators of dimension- $d, \mathscr{O}_{k}^{(d)}(k=1,2, \ldots)$, defined as $\mathscr{O}_{1}^{(3)} \equiv \bar{q} h \gamma_{5} h_{v},\left\{\mathscr{O}_{k}^{(4)}\right\} \equiv\left\{\bar{q}(\right.$ in $\left.\cdot \overleftarrow{D}) h \gamma_{5} h_{v}, \bar{q}(i v \cdot \overleftarrow{D}) h \gamma_{5} h_{v}\right\}$, and $\left\{\mathscr{O}_{k}^{(5)}\right\} \equiv\left\{\bar{q}(\text { in } \cdot \overleftarrow{D})^{2} \not h \gamma_{5} h_{v}\right.$ $\bar{q}(i v \cdot \overleftarrow{D})(i n \cdot \overleftarrow{D}) \not h \gamma_{5} h_{v}, \bar{q}(i v \cdot \overleftarrow{D})^{2} \not h \gamma_{5} h_{v}, \bar{q} i g G_{\alpha \beta} v^{\alpha} n^{\beta} \not h \gamma_{5} h_{v}, \bar{q} i g G_{\alpha \beta} \gamma^{\alpha} n^{\beta} \not \vec{h} \gamma_{5} h_{v}, \bar{q} i g G_{\alpha \beta} \gamma^{\alpha} v^{\beta} \not \vec{h} \gamma_{5} h_{v}$, $\left.\bar{q} g G_{\alpha \beta} \sigma^{\alpha \beta} \not h \gamma_{5} h_{v}\right\}$, with another light-like vector, $\bar{n}^{2}=0$, as $v_{\mu}=\left(n_{\mu}+\bar{n}_{\mu}\right) / 2$. The NLO Wilson coefficients are obtained as

$$
\begin{aligned}
& C_{1}^{(3)}(t, \mu)=1-\frac{\alpha_{s} C_{F}}{4 \pi}\left(2 L^{2}+2 L+\frac{5 \pi^{2}}{12}\right), C_{1}^{(4)}(t, \mu)=-i t\left[1-\frac{\alpha_{s} C_{F}}{4 \pi}\left(2 L^{2}+L+\frac{5 \pi^{2}}{12}\right)\right], \\
& C_{2}^{(4)}(t, \mu)=\frac{i t \alpha_{s} C_{F}}{4 \pi}(4 L-3), C_{1}^{(5)}(t, \mu)=-\frac{t^{2}}{2}\left[1-\frac{\alpha_{s} C_{F}}{4 \pi}\left(2 L^{2}+\frac{2}{3} L+\frac{5 \pi^{2}}{12}\right)\right]
\end{aligned}
$$


and, for the explicit form of $C_{2}^{(5)}(t, \mu), C_{3}^{(5)}(t, \mu), \ldots, C_{7}^{(5)}(t, \mu)$, we refer the readers to [7]. Here and below, $\mu$ is the $\overline{\mathrm{MS}}$ scale, and $\alpha_{s} \equiv \alpha_{s}(\mu)$. The double logarithm $L^{2}$ in the coefficient functions originates from the cusp singularity (see (2)). The 1-loop corrections for the 2-point function induce all of the above ten operators using the EOM, while those for the 3-point function induce only $\mathscr{O}_{4,5,6,7}^{(5)}$ associated with the field-strength tensor; as a result, the coefficients $C_{4,5,6,7}^{(5)}(t, \mu)$ involve the terms proportional to the color factor $C_{G}=N_{c}$ as well as to $C_{F}$ [7].

Taking the matrix element $\langle\cdots\rangle \equiv\langle 0|\cdots| \bar{B}(v)\rangle$ of (3), we can derive the OPE form of the $B$-meson LCDA (1). The matrix elements of the local operators in (3) are known to be related to a few nonperturbative parameters in the HQET, using the EOM and heavy-quark symmetry as demonstrated in [4, 5]: $\left\langle\mathscr{O}_{1}^{(4)}\right\rangle=4 i F(\mu) \bar{\Lambda} / 3,\left\langle\mathscr{O}_{2}^{(4)}\right\rangle=i F(\mu) \bar{\Lambda}$, with $F$ of (1) and $\bar{\Lambda}=m_{B}-m_{b}$, representing the mass difference between the $B$-meson and $b$-quark, and all seven matrix elements $\left\langle\mathscr{O}_{k}^{(5)}\right\rangle$ for the dimension-5 operators can be expressed by $F, \bar{\Lambda}$ and two additional HQET parameters $\lambda_{E}$ and $\lambda_{H}$, which are associated with the chromoelectric and chromomagnetic fields inside the $B$ meson as $\left\langle\bar{q} g \boldsymbol{E} \cdot \boldsymbol{\alpha} \gamma_{5} h_{v}\right\rangle=F(\mu) \lambda_{E}^{2}(\mu)$ and $\left\langle\bar{q} g \boldsymbol{H} \cdot \boldsymbol{\sigma} \gamma_{5} h_{v}\right\rangle=i F(\mu) \lambda_{H}^{2}(\mu)$, respectively, in the rest frame where $v=(1, \mathbf{0})$. As a result, we obtain [7] the OPE form for the LCDA (1),

$$
\begin{gathered}
\tilde{\phi}_{+}(t, \mu)=1-\frac{\alpha_{s} C_{F}}{4 \pi}\left(2 L^{2}+2 L+\frac{5 \pi^{2}}{12}\right)-i t \frac{4 \bar{\Lambda}}{3}\left[1-\frac{\alpha_{s} C_{F}}{4 \pi}\left(2 L^{2}+4 L-\frac{9}{4}+\frac{5 \pi^{2}}{12}\right)\right] \\
-t^{2} \bar{\Lambda}^{2}\left[1-\frac{\alpha_{s} C_{F}}{4 \pi}\left(2 L^{2}+\frac{16}{3} L-\frac{35}{9}+\frac{5 \pi^{2}}{12}\right)\right]-\frac{t^{2} \lambda_{E}^{2}(\mu)}{3}\left[1-\frac{\alpha_{s} C_{F}}{4 \pi}\left(2 L^{2}+2 L-\frac{2}{3}+\frac{5 \pi^{2}}{12}\right)\right. \\
\left.+\frac{\alpha_{s} C_{G}}{4 \pi}\left(\frac{3}{4} L-\frac{1}{2}\right)\right]-\frac{t^{2} \lambda_{H}^{2}(\mu)}{6}\left[1-\frac{\alpha_{s} C_{F}}{4 \pi}\left(2 L^{2}+\frac{2}{3}+\frac{5 \pi^{2}}{12}\right)-\frac{\alpha_{s} C_{G}}{8 \pi}(L-1)\right],
\end{gathered}
$$

which takes into account the Wilson coefficients to $O\left(\alpha_{s}\right)$ and a complete set of the local operators of dimension $d \leq 5$. Fourier transforming to the momentum representation and taking the first two $(j=0,1)$ regularized-moments, $M_{j}=\int_{0}^{\Lambda_{U V}} d \omega \omega^{j} \phi_{+}(\omega, \mu)$, the contributions from the first line in (5), associated with matrix elements of the dimension-3 and -4 operators, coincide completely with the result obtained in [9]. The second and third lines in (5) are generated from the dimension-5 operators. Our OPE result (5) "merges" the UV [6] and IR structures [5] peculiar to the $B$-meson LCDA, so that it embodies novel behaviors that are completely different from those of the pion LCDA: $\mu$ and $t$ are strongly correlated due to the logarithmic contributions, $L=\ln \left[i(t-i 0) \mu e^{\gamma_{E}}\right]$, from radiative corrections, so that the DA is not Taylor expandable about $t=0$, which in turn implies the UV divergence in the moments [4, 8, 9], $M_{j} \rightarrow \infty$ as $\Lambda_{U V} \rightarrow \infty$. The DA receives the contributions from (many) higher dimensional operators, in particular, from those associated with the long-distance gluon fields inside the $B$-meson. It is instructive to draw a comparison with the previous results, concerning UV or IR structure: one can prove [7] that (5) satisfies the renormalization group equation for (1), which is governed by the evolution kernel [6] determined by the (single) UV poles in (2). On the other hand, (5) reveals that the solution of the EOM constraints for (1), which was obtained in [5], is subject to additional effects from radiative corrections, see [7] for the detail (see also [8]). Such corrections to the EOM constraints at order $\alpha_{s}$ in perturbation theory is peculiar to the heavy-meson LCDAs in the HQET and does not arise for the case of the (higher twist) LCDAs for the light mesons, $\pi, \rho$, etc. [11].

Our OPE form (5) allows us to parameterize all nonperturbative contributions in the $B$-meson LCDA (1) by three HQET parameters, $\bar{\Lambda}, \lambda_{E}$ and $\lambda_{H}$, and gives a model-independent description 

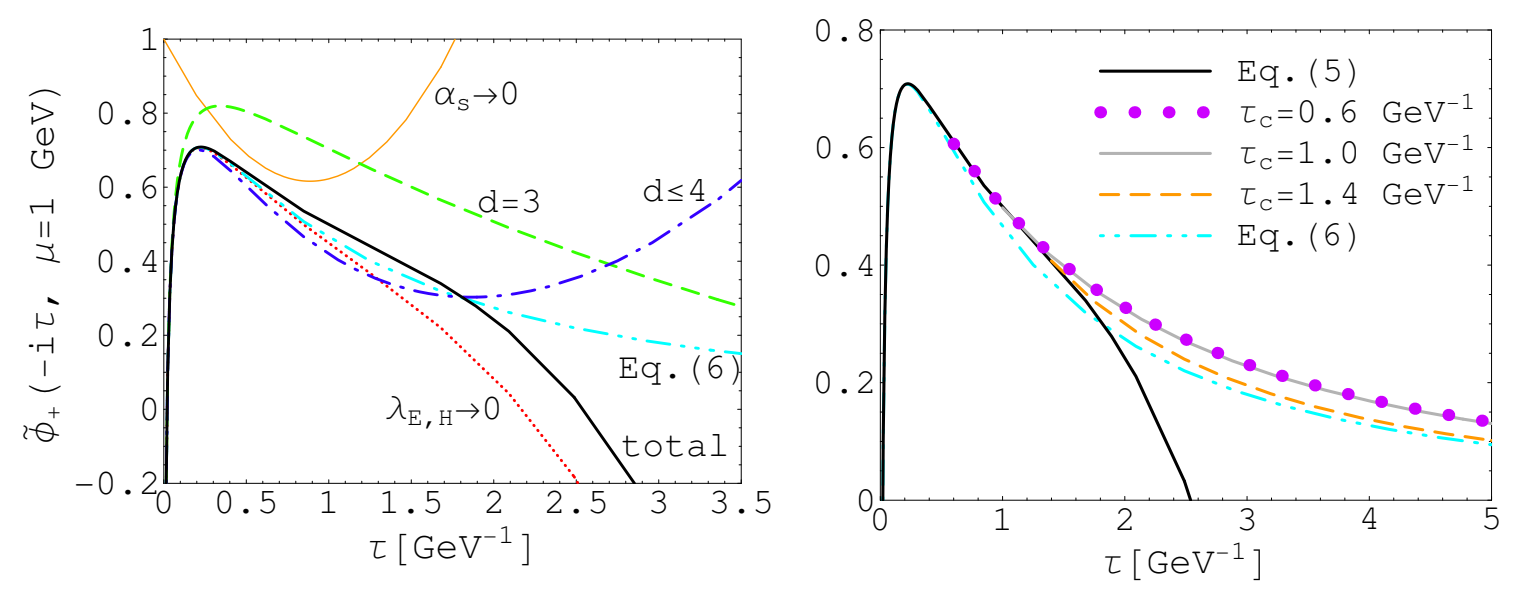

Figure 1: The $B$-meson LCDA at $\mu=1 \mathrm{GeV}$ using the OPE (left) and its continuation with a model (right).

of the $B$-meson LCDA when $t \lesssim 1 / \mu\left(\leq 1 / \Lambda_{\mathrm{QCD}}\right)$, taking into account the UV and IR structures simultaneously. Here, we evaluate (5) at the scale $\mu=1 \mathrm{GeV}: \bar{\Lambda}=m_{B}-m_{b}$ in (5) is defined by the $b$ quark pole mass $m_{b}$. Following [9], we eliminate $\bar{\Lambda}$ in favor of a short-distance parameter, $\bar{\Lambda}_{D A}$, free from IR renormalon ambiguities and written as $\bar{\Lambda}=\bar{\Lambda}_{D A}(\mu)\left[1+(7 / 16 \pi) C_{F} \alpha_{s}\right]-(9 / 8 \pi) \mu C_{F} \alpha_{s}$, to one-loop accuracy; $\bar{\Lambda}_{D A}(\mu)$ can be related to another short-distance mass parameter whose value is extracted from analysis of the spectra in inclusive decays $B \rightarrow X_{s} \gamma$ and $B \rightarrow X_{u} l \nu$, leading to $\bar{\Lambda}_{D A}(\mu=1 \mathrm{GeV}) \simeq 0.52 \mathrm{GeV}$ [9]. For the novel parameters associated with the dimension-5 operators, we use the central values of $\lambda_{E}^{2}(\mu)=0.11 \pm 0.06 \mathrm{GeV}^{2}, \lambda_{H}^{2}(\mu)=0.18 \pm 0.07 \mathrm{GeV}^{2}$, at $\mu=1 \mathrm{GeV}$, which were obtained by QCD sum rules [4]; no other estimate exists for $\lambda_{E}$ or $\lambda_{H}$. We calculate (5) for imaginary LC separation, performing the Wick rotation $t \rightarrow-i \tau$ [4, 8].

The results for $\tilde{\phi}_{+}(-i \tau, \mu=1 \mathrm{GeV})$ using (5) are shown as a function of $\tau$ in the LHS of Fig. 1 [7]: the wide-solid curve shows the whole contributions of (5), while the narrow-solid curve shows the result for $\alpha_{s} \rightarrow 0$; the NLO perturbative corrections are at the 10-30\% level for moderate $\tau$ of order $1 \mathrm{GeV}^{-1} \sim 1 / \mu$, while they are very large for $\tau \rightarrow 0$ because of singular logarithms $L^{2}$ and $L$. The dashed and dot-dashed curves show the contributions of the first two terms and the first line in (5), respectively, associated with the operators of dimension $d=3$ and $d \leq 4$, while the dotted curve gives the results of (5) when $\lambda_{E}=\lambda_{H}=0$. For moderate $\tau$, the contributions from the dimension- 4 operators suppress the DA by $30-40 \%$, but the dimension- 5 operators, in contrast, lead to enhancement by $10-20 \%$ with significant effects from $\lambda_{E}$ and $\lambda_{H}$. Our $B$-meson LCDA (5) indeed works up to moderate LC distances $\tau$, where the hierarchy among the dashed, dot-dashed, and wide-solid curves demonstrates convergence of the OPE (3).

The two-dot-dashed curve in the LHS of Fig. 1 shows the behavior of the two-component ansatz by Lee and Neubert [9], which is given in momentum space as

$$
\phi_{+}^{\mathrm{LN}}(\omega, \mu)=N \frac{\omega}{\omega_{0}^{2}} e^{-\omega / \omega_{0}}+\theta\left(\omega-\omega_{t}\right) \frac{C_{F} \alpha_{s}}{\pi \omega}\left[\left(\frac{1}{2}-\ln \frac{\omega}{\mu}\right)+\frac{4 \bar{\Lambda}_{D A}}{3 \omega}\left(2-\ln \frac{\omega}{\mu}\right)\right],
$$

where the second term reproduces the correct asymptotic behavior of the DA (1) for $\omega \gg \Lambda_{\mathrm{QCD}}$ and the first term represents the nonperturbative component modeled by an exponential form [4], with $\omega_{t}=2.33 \mathrm{GeV}, N=0.963$, and $\omega_{0}=0.438 \mathrm{GeV}$ at $\mu=1 \mathrm{GeV}$; these parameters are fixed by match- 


\begin{tabular}{|c|c|c|c||c|c|c|}
\hline & \multicolumn{2}{|c||}{$\lambda_{E}^{2}=0.11 \mathrm{GeV}^{2}, \lambda_{H}^{2}=0.18 \mathrm{GeV}^{2}$} & \multicolumn{3}{c|}{$\lambda_{E}^{2}=\lambda_{H}^{2}=0$} \\
\hline$\tau_{c}\left[\mathrm{GeV}^{-1}\right]$ & $N$ & $\omega_{0}[\mathrm{GeV}]$ & $\lambda_{B}^{-1}\left[\mathrm{GeV}^{-1}\right]$ & $N$ & $\omega_{0}[\mathrm{GeV}]$ & $\lambda_{B}^{-1}\left[\mathrm{GeV}^{-1}\right]$ \\
\hline 0.4 & 0.816 & 0.257 & $3.11(0.23+2.88)$ & 0.832 & 0.301 & $2.69(0.23+2.46)$ \\
0.6 & 0.850 & 0.306 & $2.70(0.35+2.35)$ & 0.899 & 0.394 & $2.19(0.35+1.84)$ \\
0.8 & 0.852 & 0.308 & $2.69(0.47+2.22)$ & 0.966 & 0.461 & $1.99(0.46+1.53)$ \\
1.0 & 0.858 & 0.313 & $2.66(0.58+2.08)$ & 1.11 & 0.572 & $1.79(0.56+1.23)$ \\
1.2 & 0.910 & 0.349 & $2.51(0.67+1.84)$ & 1.55 & 0.839 & $1.56(0.64+0.92)$ \\
1.4 & 1.09 & 0.456 & $2.22(0.76+1.46)$ & 4.43 & 1.95 & $1.32(0.71+0.61)$ \\
1.6 & 1.81 & 0.777 & $1.87(0.83+1.04)$ & 9.82 & -4.55 & $1.11(0.77+0.34)$ \\
\hline
\end{tabular}

Table 1: Parameters of the model function $N /\left(\tau \omega_{0}+1\right)^{2}$ for $\tau \geq \tau_{c}$ with different values of $\tau_{c}$, and the results of the inverse moment $\lambda_{B}^{-1}(\mu)$ at $\mu=1 \mathrm{GeV}$, with the first and second numbers in the parentheses denoting the contributions from the first and the second terms in the RHS of (7).

ing the first two $(j=0,1)$ cut-moments $\int_{0}^{\Lambda_{U V}} d \omega \omega^{j} \phi_{+}^{\mathrm{LN}}(\omega, \mu)$ with the OPE for the corresponding cut-moments $M_{0,1}$ derived in [9], where the operators of dimension $d \leq 4$ and the corresponding Wilson coefficients at NLO are taken into account. For $\tau \lesssim 1 \mathrm{GeV}^{-1}$, the Lee-Neubert ansatz (6) shows behavior similar to (5) with $\lambda_{E}=\lambda_{H}=0$ substituted; note that the first term of (6) produces particular contributions associated with the operators of dimension- 5 and higher.

For $\tau \gg 1 \mathrm{GeV}^{-1}$, the contributions associated with higher-dimensional operators become important, and the OPE diverges (see (5) and Fig. 1); thus, one has to rely on a certain model for the large $\tau$ behavior and connect the model-independent descriptions at small and moderate $\tau$ to that model. The results in Fig. 1 suggest the possibility of connecting the behavior for $\tau \leq \tau_{c}\left(\tau_{c} \sim 1 \mathrm{GeV}^{-1}\right)$ given by our OPE form (5) to that for $\tau \geq \tau_{c}$, given by the coordinatespace representation of the first term of (6), $\int_{0}^{\infty} d \omega e^{-\omega \tau}\left(N \omega / \omega_{0}^{2}\right) e^{-\omega / \omega_{0}}=N /\left(\tau \omega_{0}+1\right)^{2}$. Here, $N$ and $\omega_{0}$ can be determined such that both the resulting total DA $\tilde{\phi}_{+}(-i \tau, \mu)$ and its derivative $\partial \tilde{\phi}_{+}(-i \tau, \mu) / \partial \tau$ are continuous at $\tau=\tau_{c}$. In the LHS of Table 1, we show [7] the values of $N$ and $\omega_{0}$ obtained by solving the corresponding conditions of the continuity for $\mu=1 \mathrm{GeV}$. (The RHS of Table 1 shows the results that would be obtained by solving the similar continuity conditions with $\lambda_{E}=\lambda_{H}=0$.) In the RHS of Fig. 1, the wide-solid and two-dot-dashed curves are same as those in the LHS, and the dotted, solid-gray, and dashed curves show the behavior of the above model function $N /\left(\tau \omega_{0}+1\right)^{2}$ for $\tau \geq \tau_{c}$ with $\tau_{c}=0.6,1.0$, and $1.4 \mathrm{GeV}^{-1}$, respectively, using the corresponding values of $N$ and $\omega_{0}$ in the LHS of Table 1; these three curves behave as $\sim N /\left(\omega_{0}^{2} \tau^{2}\right)$ at large $\tau$, with larger $N / \omega_{0}^{2}$ than those of (6) and the RHS of Table 1. Indeed, we can show that $N / \omega_{0}^{2}=\left(9 / 4 \bar{\Lambda}_{D A}^{2}\right)\left\{1+\tau_{c} \bar{\Lambda}_{D A}\left[\lambda_{E}^{2} / \bar{\Lambda}_{D A}^{2}+\lambda_{H}^{2} /\left(2 \bar{\Lambda}_{D A}^{2}\right)-1\right]\right\}+\cdots$, using the continuity of $\tilde{\phi}_{+}(-i \tau, \mu), \partial \tilde{\phi}_{+}(-i \tau, \mu) / \partial \tau$ at $\tau=\tau_{c}$, and thus the contributions of $\lambda_{E}$ and $\lambda_{H}$ enhance $N / \omega_{0}^{2}$.

Using these results, we calculate the first inverse moment of the LCDA,

$$
\lambda_{B}^{-1}(\mu)=\int_{0}^{\infty} d \omega \frac{\phi_{+}(\omega, \mu)}{\omega}=\int_{0}^{\tau_{c}} d \tau \tilde{\phi}_{+}(-i \tau, \mu)+\int_{\tau_{c}}^{\infty} d \tau \tilde{\phi}_{+}(-i \tau, \mu),
$$

which is of particular interest for the QCD description of exclusive $B$-meson decays. We substitute (5) and the model function, $N /\left(\tau \omega_{0}+1\right)^{2}$, into the first and the second terms in the RHS, respectively, and the results are shown in Table 1 for each value of $\tau_{c}$ [7]. The "stable" behavior observed for $0.6 \mathrm{GeV}^{-1} \lesssim \tau_{c} \lesssim 1 \mathrm{GeV}^{-1}$ in the LHS of Table 1 and in the RHS of Fig. 1 
suggests that $\lambda_{B}^{-1}(\mu=1 \mathrm{GeV}) \simeq 2.7 \mathrm{GeV}^{-1}$, i.e., $\lambda_{B}(\mu=1 \mathrm{GeV}) \simeq 0.37 \mathrm{GeV}$. This value of $\lambda_{B}$ is somewhat smaller than the previous estimates that include nonperturbative and/or perturbative QCD corrections [8, 9, 12] (e.g., (6) gives $\lambda_{B}(\mu=1 \mathrm{GeV}) \simeq 0.48 \mathrm{GeV}$ ). A value of $\lambda_{B}$ that is as small as our value was adopted in [1]. Note that in the RHS of Table 1 with $\lambda_{E, H}=0$, the stable behavior is not seen as clearly as in the LHS, and $\lambda_{B}$ assumes larger values than in the latter. These results demonstrate that the novel HQET parameters, $\lambda_{E}$ and $\lambda_{H}$, associated with the dimension-5 quark-antiquark-gluon operators, could lead to smaller value of $\lambda_{B}$. In particular, using the values $\lambda_{E}^{2}=0.17 \mathrm{GeV}^{2}, \lambda_{H}^{2}=0.25 \mathrm{GeV}^{2}$, which correspond to their upper bound from the QCD sum rule estimate at $\mu=1 \mathrm{GeV}$ [4], we find that the wide-solid curve in Fig. 1 becomes further enhanced in the moderate $\tau$ region, so that (7) gives $\lambda_{B}(\mu=1 \mathrm{GeV}) \sim 0.2 \mathrm{GeV}$ or smaller.

To summarize, we have derived the OPE that embodies both the notorious UV and IR behaviors of the $B$-meson LCDA, including all contributions from the local operators of dimension $d \leq 5$ and the corresponding Wilson coefficients at NLO accuracy. This OPE provides us with the most accurate description of the $B$-meson LCDA for distances less than $\sim 1 / \Lambda_{\mathrm{QCD}}$. We have also used the model-independent behaviors from our OPE to constrain the long-distance behavior of the LCDA and estimate the first inverse moment $\lambda_{B}^{-1}$. The results demonstrated the impact of the novel HQET parameters, associated with the dimension-5 quark-antiquark-gluon operators.

\section{References}

[1] M. Beneke et al., Nucl. Phys. B591 (2000) 313; B606 (2001) 245; M. Beneke and M. Neubert, Nucl. Phys. B675 (2003) 333; M. Beneke and S. Jager, Nucl. Phys. B751 (2006) 160; B768 (2007) 51.

[2] C. W. Bauer, D. Pirjol and I. W. Stewart, Phys. Rev. Lett. 87 (2001) 201806; Phys. Rev. D67 (2003) 071502; H. n. Li and H. L. Yu, Phys. Rev. Lett. 74 (1995) 4388; Phys. Rev. D53 (1996) 2480.

[3] C. W. Bauer et al., Phys. Rev. D70 (2004) 054015; N. Kivel, JHEP 0705 (2007) 019; V. Pilipp, Nucl. Phys. B794 (2008) 154; G. Bell, Nucl. Phys. B795 (2008) 1; arXiv:0902.1915 [hep-ph].

[4] A. G. Grozin and M. Neubert, Phys. Rev. D55 (1997) 272; M. Beneke and T. Feldmann, Nucl. Phys. B592 (2001) 3; A. Khodjamirian, T. Mannel and N. Offen, Phys. Rev. D75 (2007) 054013.

[5] H. Kawamura, J. Kodaira, C.F. Qiao and K. Tanaka, Phys. Lett. B523 (2001) 111; Erratum-ibid. B536 (2002) 344; Mod. Phys. Lett. A18 (2003) 799; Nucl. Phys. B (Proc. Suppl.) 116 (2003) 269.

[6] B. O. Lange and M. Neubert, Phys. Rev. Lett. 91 (2003) 102001; S. Descotes-Genon and N. Offen, arXiv:0903.0790 [hep-ph]; arXiv:0904.4687 [hep-ph].

[7] H. Kawamura and K. Tanaka, Phys. Lett. B673 (2009) 201.

[8] V. M. Braun, D. Y. Ivanov and G. P. Korchemsky, Phys. Rev. D69 (2004) 034014; G. Bell and T. Feldmann, JHEP 0804 (2008) 061.

[9] S. J. Lee and M. Neubert, Phys. Rev. D72 (2005) 094028.

[10] J. S. Schwinger, Phys. Rev. 82 (1951) 664; E. V. Shuryak and A. I. Vainshtein, Nucl. Phys. B199 (1982) 451; B201 (1982) 141; I. I. Balitsky and V. M. Braun, Nucl. Phys. B311 (1989) 541.

[11] V. M. Braun and I. E. Filyanov, Z. Phys. C48 (1990) 239; P. Ball, V. M. Braun, Y. Koike and K. Tanaka, Nucl. Phys. B529 (1998) 323; P. Ball and V. M. Braun, Nucl. Phys. B543 (1999) 201.

[12] P. Ball and E. Kou, JHEP 0304 (2003) 029; A. Khodjamirian, T. Mannel and N. Offen, Phys. Lett. B620 (2005) 52. 